\title{
RELACIONES CENTRO-PENINSULARES DURANTE EL PERIODO CLÁSICO TARDÍO
}

\author{
Lorraine A. Williams-Beck
}

Universidad Autónoma de Campeche

\begin{abstract}
ABstracr: Formal patio group analyses from twenty three archaeological sites in the Chenes Region identify certain architectural and spatial homologies among sites within the Central and Northwestern Yucatan Peninsula area. Chenes ceramics analyses not only confirm George Brainerd's hypothesis regarding regional and temporal variation for Medium Slatewares produced in Yucatan Peninsula, but also emphasize similar formal repertoires and paste composition between Late Classic regional polychrome traditions and contemporary pre-Cehpech Medium Slatewares. These data also suggest that innovative ceramic techniques began in the Central Peninsular area during the late phase of the Early Classic Period may have inspired pre-Cehpech monochrome slateware production that would later replace regional polychrome traditions throughout the greater Northern Lowlands. Finally, shared ceramic and architectural spatial similarities in this area points to possible social and political conditions prevailing in certain areas during the Late and Terminal Classic Periods, and that these conditions may have been adopted, slightly modified, and reproduced in important Postclassic urban centers, such as Chichen Itza and Mayapan.
\end{abstract}

Resumen: Los análisis de conjuntos de patios formales en veintitrés sitios en la región de los Chenes identifican ciertos elementos homólogos en la arquitectura y los espacios culturales entre la zona centro y noroeste de la península de Yucatán. El análisis de la cerámica de la región de los Chenes no sólo sustenta la hipótesis de George Brainerd sobre las variaciones regionales y temporales en la Pizarra Mediana producida en la península, sino también hace hincapié en las similitudes que existen entre los repertorios formales y en la composición de la pasta para las tradiciones policromas regionales y las Pizarras Medianas pre-Cehpech. Estos datos sugieren asimismo que las técnicas cerámicas innovadoras que aparecieron durante la última fase del periodo Clásico Temprano pudiesen haber inspirado la producción de la cerámica Pizarra Mediana pre-Cehpech, que reemplazaria las tradiciones regionales de cerámica policroma a través de las tierras bajas del norte en la península de Yucatán. Finalmente, las similitudes entre las tradiciones arquitectónicas y cerámicas compartidas en la región centro-peninsular sugieren evidencias sobre ciertos sistemas políticos y sociales que prevalecieron en algunas áreas durante los periodos Clásico Tardío y Clásico Terminal y que estas formas de organización pudieran haber sido adoptadas, modificadas y reproducidas más tarde en algunos centros urbanos principales del periodo Posclásico, como Chichén Itzá y Mayapán.

Una meta que los estudios de la cultura maya en la península de Yucatán quieren alcanzar es reconstruir momentos históricos en la vida de los pueblos prehispánicos. Si bien los documentos del siglo xv hacen referencia a algunos de ellos asentados en la zona litoral y el área norte peninsular, el devenir cultural de otros grupos que vivían tierra adentro y que no aparecen en estas fuentes descansa en el análisis y la interpretación de las evidencias arqueológi-

Estudios de Cultura Maya. Vol. XXI, 2000

Instituto de Investigaciones Filológicas/

Centro de Estudios Mayas, UNAM

ISSN: 0185-2574 
cas, arquitectónicas y de la distribución de éstas en el espacio cultural. Por ello, las huellas dejadas por estos pueblos forman una parte medular de las herramientas de trabajo para rescatar el sentido de cómo era su vida y para poder calcular cuáles fueron las relaciones que tuvieron con otros pueblos en el área maya. Sin embargo, escoger elementos aislados del registro arqueológico para recontruir la historia de los grupos mayances que habitaban la "tierra de nadie" en el centro de la península antes del siglo xvi, sólo proporciona pistas fragmentarias de cómo eran sus instituciones sociales, políticas, culturales y económicas. Por ello, hay que considerar aquellos elementos en su conjunto, a través de contextos arqueológicos y arquitectónicos que se repiten en el paisaje, para poder brindar un panorama más amplio sobre las relaciones que mantenían con otros grupos a su alrededor.

Reconstruir la historia de cualquier pueblo implica buscar las evidencias a través de los restos materiales que sugieren algunas relaciones con otros en el espacio y el tiempo. A menudo este marco de referencia proporciona información sobre el intercambio de ideas, bienes y estructuras institucionales de carácter político, económico y social. Con el avance de estudios científicos dirigidos a esclarecer problemas específicos en la península de Yucatán (figura 1), la compleja historia de los pueblos prehispánicos asentados ahí parece desenredarse cada vez más, porque se comprueban algunas Estudios de Cultura Maya. Vol

Instituto de Investigaciones Filológicas/

Centro de Estudios Mayas, UNAM hipótesis propuestas anteriormente o, en su defecto, se deben plantear otras a raíz de la información nueva.

A los casi 10 lustros de investigación sobre vestigios culturales en Edzná y otros lugares prehispánicos en el estado de Campeche, estos esfuerzos vislumbran otras piezas del cuadro sobre las relaciones que este sitio tenía con otros pueblos en su alrededor. Nuestros colegas Alberto Ruz, Raúl Pavón Abreu, Román Piña Chan y George F. Andrews fueron los pioneros en despertar el interés sobre la gran ciudad de Edzná. Sus resultados se suman a otros trabajos recientes en Edzná que destacan su papel en el devenir histórico del área maya. Sin embargo, esto dificilmente se comprendería mejor si no se considerara el contexto macrorregional en que se encontraba Edzná con sus pares de la zona centro-peninsular.

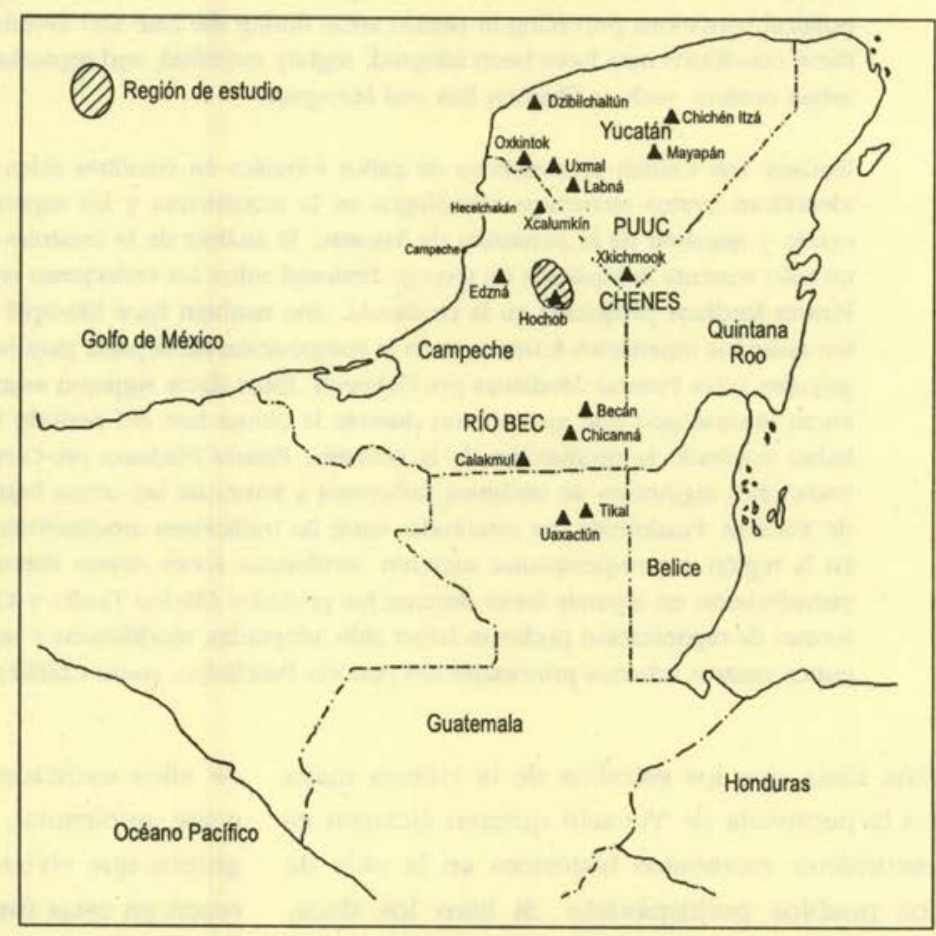

Figura 1. Área maya peninsular 
Un área en particular que queda a unos $40 \mathrm{~km}$ al oriente de Edzná es la región de los Chenes. Sus sitios muestran espléndidos vestigios arqueológicos y arquitectónicos que nos permiten inferir un apogeo cultural sin precedentes a partir del año 550 de nuestra era, durante el periodo Floreciente (Brainerd 1958) u horizontes culturales conocidos ahora como el Clásico Tardío y el Clásico Terminal (Ball, 1977; Boucher, 1990; Forsyth, 1983; Williams-Beck, 1993, 1994, 1999).

El presente ensayo pretende ofrecer algunas interpretaciones nuevas sobre este corto periodo en el desarrollo histórico cultural de los pueblos prehispánicos del área centro-peninsular. Los resultados de los análisis arquitectónicos, cerámicos y de los espacios urbanos del área de los Chenes por quien esto escribe aportan elementos nuevos que sugieren una estrecha relación entre los Chenes, el sitio de Edzná y otros lugares en la península yucateca desde el Clásico Tardio. Asimismo, este vínculo parece haber continuado hasta el Clásico Terminal, es decir desde 550 hasta aproximadamente el 1000 o el 1100 de nuestra era. A continuación se presentan algunas evidencias que describen esta relación cercana.

\section{La arquitectura}

Investigadores de la cultura prehispánica maya estiman que la arquitectura es uno de los productos culturales que representan la mayor inversión de energía y esfuerzo para un pueblo dado (Kurjack, 1975, 1994). Siguiendo este punto de vista, hace tiempo la literatura sobre arquitectura en Edzná (Ruz Lhuillier, 1945; Andrews, 1968, 1975; Piña Chan, 1975, 1985; Benavides, 1997) asienta la grandeza de sus complejos arquitectónicos, cuando se comparen con otros elementos contemporáneos en sitios de las tierras bajas mayas en la península de Yucatán.

Asimismo, estos estudiosos no sólo le confie- ren un carácter único a Edzná, sino también sugieren que sus complejos arquitectónicos son un ejemplo del "estilo" propio del lugar. Ahora se sabe que esta particularidad parece haberse fundado en una división del estado de Campeche en distintas regiones ecológicas. De ahí se establecería una relación estrecha entre la cultura y el ambiente, sin cuestionar una correspondencia aparente entre la evidencia cultural y el medio natural en este y otros lugares prehispánicos. Si bien hace falta realizar estudios arqueológicos y arquitectónicos de mayor alcance respecto a extensiones geográficas y territoriales en el área maya y más aún en la zona centropeninsular, los fundamentos para poder afirmar esta relación entre el desarrollo cultural y su respuesta en el medio natural están por esclarecerse todavía.

A pesar de que esta idea de regiones ecológicas equivalentes a áreas culturales en el área maya cobraría seguidores en otros estudios posteriores, Alberto Ruz Lhuillier (1945: 89) fue el primero en reconocer el intercambio de ideas entre distintas zonas de las tierras bajas que se plasmarían en la arquitectura, independientemente del nicho ecológico en que se desenvolvieran, cuando señaló algunas relaciones culturales que Edzná pudiera haber tenido con otros sitios cercanos. Por su experiencia y anos de trabajo en este lugar, Piña Chan opinaría después que "...quizá sería más propio considerarlo como una combinación de varios atributos arquitectónicos, provenientes tanto de las áreas culturales del sur [Petén], así como de las del norte [Puuc]" (1985: 27; Andrews, 1984: 75).

Si bien la Estructura de los Cinco Pisos que corona la Gran Acrópolis muestra una arquitectura monumental grandiosa $y$, tal vez, única en las tierras bajas mayas, ahora no se le considera como un "estilo" arquitectónico particular. Estudios recientes resaltan que dificilmente se comprendería el concepto de estilo sin hacer un 
análisis detallado de la secuencia arquitectónica del lugar, incluyendo las fases constructivas y los materiales cerámicos asociados con los contextos urbanos (Carrasco y Boucher, 1986: 92).

De acuerdo con estos nuevos planteamientos, la arquitectura en Edzná y en la región de los Chenes parece mostrar ciertos detalles de diseño básico, en los cuales se refleja una armonía de conceptos durante el auge de construcción del Clásico Tardío en ambos lugares (Andrews, comunicación personal, 1990). En cambio, los conjuntos arquitectónicos que contienen mascarones modelados en estuco y basamentos piramidales con esquinas inferiores redondeadas $^{1}$ muestran cierto sazón sureño (Andrews, 1984: 75). Enseguida del Clásico Tardío, otros conjuntos arquitectónicos en el sitio suelen ilustrar algunos elementos provenientes de las zonas

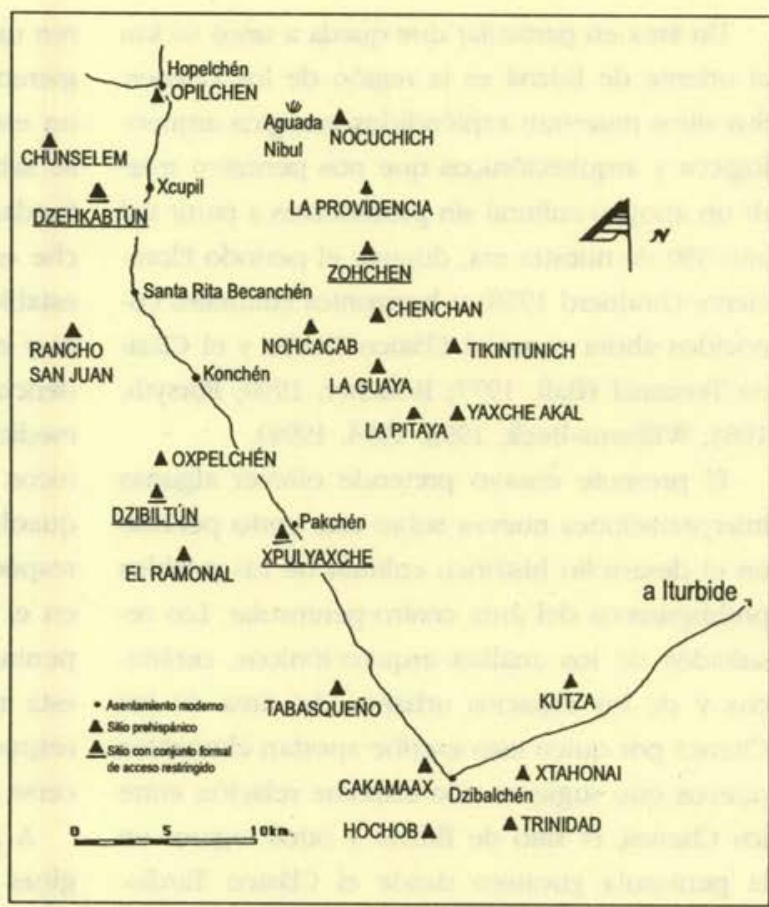

Figura 2. Mapa del área de estudio en la región de los Chenes Puuc y Chenes. ${ }^{2}$ Además de éstos se pueden señalar otros elementos constructivos que fueron compartidos entre Edzná y los Chenes: las escalinatas de bloque monolítico, ${ }^{3}$ ubicadas en la plataforma de la Gran Acrópolis y en la estructura 3 del complejo XI (la Pequeña Acrópolis). Las mismas escalinatas aparecen también en la arquitectura monumental de sitios como Dzehkabtún, Pakchén (Xpulyaxché), Dzibiltún,

Trinidad y Tik'intunich en la región de los Chenes (figura 2), así como en Dzibilchaltún, Aké e Izamal en las tierras bajas del norte. Si bien se comparten algunos elementos constructivos y motivos decorativos que reflejan el intercambio de ideas entre Edzná y la región de los Chenes, los mismos elementos suelen presentarse también en el área Puuc u otros lugares mayas durante la misma época. ${ }^{4}$ Por ello, el análi-

' La temporalidad de construcción para estos elementos parece ser previa a este periodo; véase Millet Cámara, 1989: 517-521 y García Cruz, 1989: 523-530.

${ }^{2}$ Las columnas monolíticas de fuste cilindrico con marcado énfasis que adornan los vanos del lado poniente de la Estructura de los Cinco Pisos son un elemento constructivo considerado como del área Puuc; los mismos elementos con fuste cilíndrico recto en las estructuras U7-10 y U7-11 del Anexo de los Cuchillos parecen ser del área de los Chenes.

${ }^{3}$ De acuerdo con los trabajos de restauración arquitectónica realizados en 1987 por el arqueólogo Luis Millet Cámara (comunicación personal, 1990), la escalinata de bloque monolítico en la Gran Acrópolis de Edzná parece corresponder al horizonte Clásico Temprano.

${ }^{4}$ Los trabajos arqueológicos recientes en Dzibilchaltún restauraron la estructura 44, “...un edificio del tipo palacio, que cierra la Plaza Central del sitio... [con múltiples] entradas en su fachada frontal..." (Maldonado, 1993) y escalinatas de bloques monolíticos como acceso a ello. Esta estructura parece ser una réplica de la "Nohochná" (estructura número 3) ubicada al ladogorientaldde la explanada y frente a la Gran Acrópolis en Edzná.

Instituto de Investigaciones Filológicas/

Centro de Estudios Mayas, UNAM

ISSN: 0185-2574 


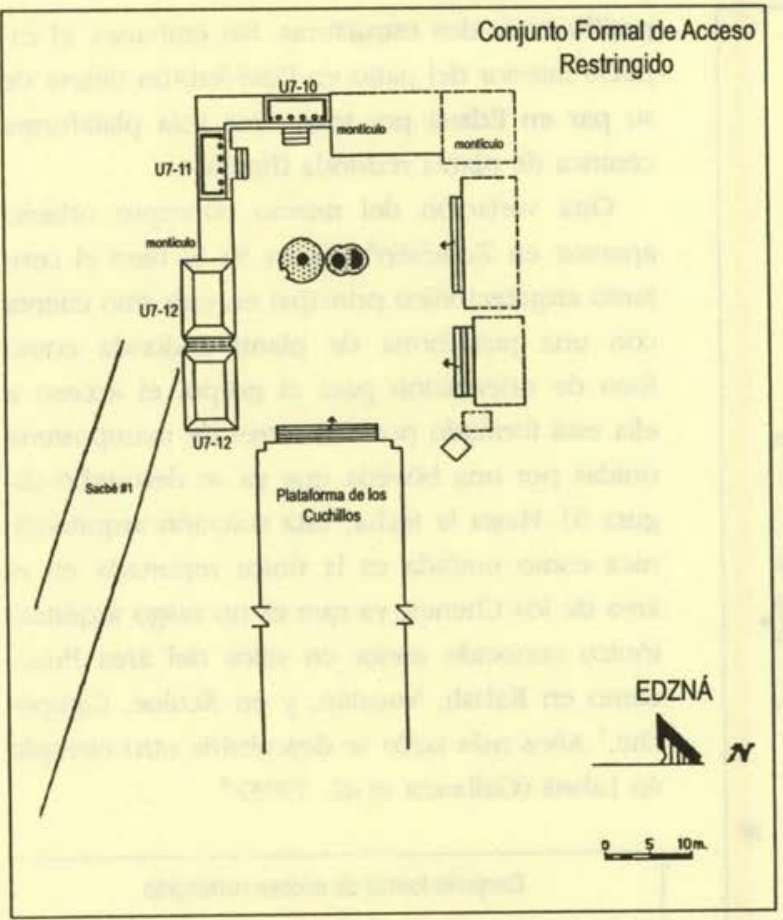

Figura 3. Anexo de los Cuchillos en Edzná

sis debería enfocarse en los espacios arquitectónicos y en el desarrollo urbano, que incluyen conjuntos de estructuras y su distribución en el tiempo y espacio, para reconstruir las relaciones derivadas del intercambio entre los sitios del área centro-peninsular.

Por desarrollo urbano se entiende un proceso de cambio cultural (Press y Smith, 1980: 1), donde el patrón arquitectónico modifica el paisaje natural para reflejar los valores, las actitudes y el comportamiento del pueblo en un área y momento histórico dados. De tal manera que una repetición en estos contextos prehispánicos mayas de tiempo y espacio nos permite formular hipótesis acerca del concepto urbano en su marco cultural compartido. De acuerdo con este punto de vista, entonces, [un]a asociación constante de variables, como el tamaño, el dominio jerárquico, la distribución de grupos de patios y la orientación de estructuras de mayor envergadura en ellos, apoya a un concepto genérico de variabilidad, con el cual aquellos [conjuntos arquitectónicos] fueron modelados. Este concepto, pero en una escala mayor... Ise comprende por la réplica estructural que presentan estos conjuntos arquitectónicos en el espaciol. Una consistencia [de distribución y de contenido] sugiere que las afinidades [arquitectónicas] de Edzná vienen de las tierras bajas mayas...[en sitios que cuentan] con un desarrollo pre-Puuc en la península de Yucatán (Andrews, 1984: 31; traducción del inglés por la autora).

Por ello, a continuación se refiere una réplica estructural específica, los conjun tos arquitectónicos de acceso restringido (Williams-Beck, 1993), que está ubicada en la zona de mayor concentración de arquitectura monumental en algunos sitios del área de los Chenes.

El primer ejemplo de esta naturaleza es el Anexo de los Cuchillos en Edzná. Este grupo es un conjunto de acceso restringido mediante una bóveda de pasillo (la estructura U7-12) que conecta el sacbé número 1 al exterior del conjunto con las estructuras distribuidas en torno a un patio o espacio compartido en el grupo. El foco de orientación (Potter, 1977) lo forman dos plataformas de pequeñas dimensiones, una de planta redonda $y$ otra, anexa a ésta, de planta ovalada (figura 3).

El grupo parece mostrar una temporalidad que va del Clásico Tardío al Posclásico Temprano, según sus elementos de construcción (Andrews, 1984; Millet Cámara, 1993) y por unos cuantos tiestos de cerámica a flor de tierra, observados por quien esto escribe. ${ }^{5}$ Si bien'Andrews (1984) y

s Se extravió el material cerámico de la excavación del conjunto en 1987, un suceso que desafortunadamente impidió un análisis detallado (Millet Cámara, comunicación personal, 1990). 


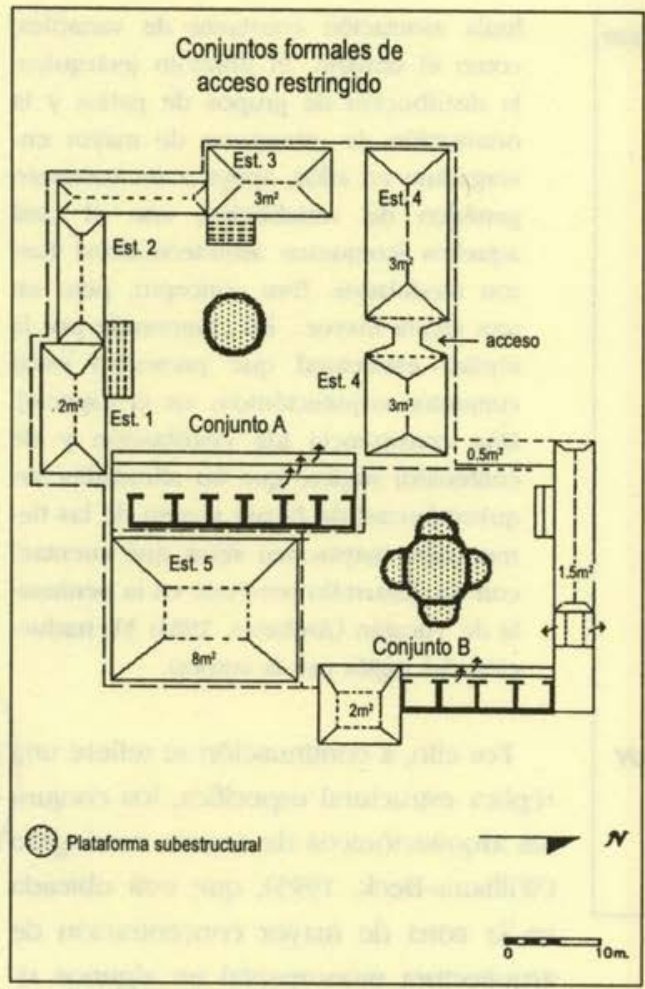

Figura 4. Conjunto de acceso restringido en Dzehkabtún

otros postulan una relación cultural arquitectónica con grupos contemporáneos del área Puuc al norte para este conjunto, a continuación se ofrecen algunas variaciones observadas en cuatro sitios en el área de los Chenes que sugieren una réplica estructural de este conjunto y sus elementos constituyentes.

Por ejemplo, el sitio de Dzehkabtún cuenta también con un grupo de patio similar (el conjunto A) en el área de mayor concentración de arquitectura monumental. En este conjunto el acceso principal está formado por una bóveda de pasillo entre dos estructuras. Sin embargo, el espacio interior del patio en Dzehkabtún difiere de su par en Edzná por tener una sola plataforma céntrica de planta redonda (figura 4).

Otra variación del mismo concepto urbano aparece en Zohchén ${ }^{6}$ (figura 5). Si bien el conjunto arquitectónico principal en este sitio cuenta con una plataforma de planta redonda como foco de orientación para el grupo, el acceso a ella está formado por dos torres de mampostería unidas por una bóveda que ya se derrumbó (figura 6). Hasta la fecha, esta solución arquitectónica como entrada es la única reportada en el área de los Chenes, ya que es un rasgo arquitectónico conocido mejor en sitios del área Puuc, como en Kabah, Yucatán, y en Xculoc, Campeche. ${ }^{7}$ Años más tarde se descubriría otro ejemplo en Labná (Gallareta et al., 1993). ${ }^{8}$

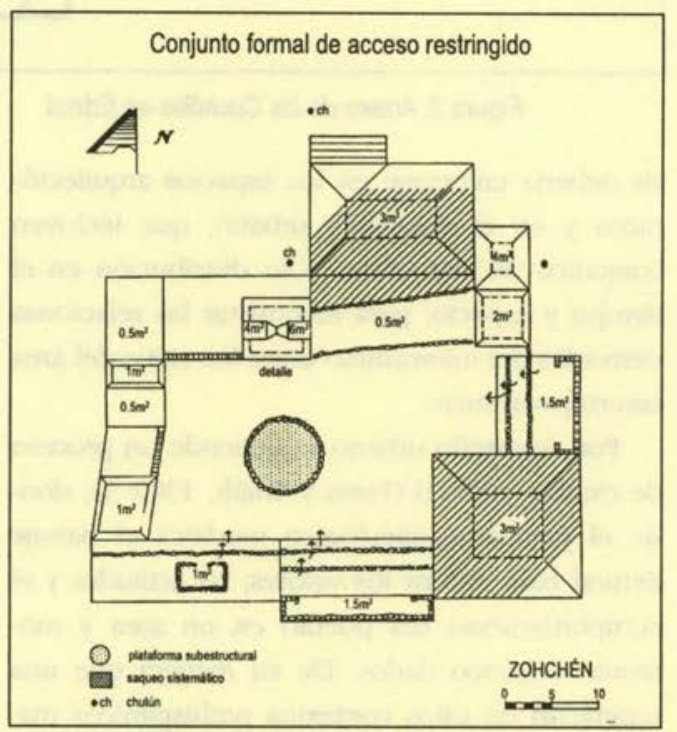

Figura 5. Conjunto de acceso restringido en Zohchén

\footnotetext{
${ }^{6}$ Andrews (1988a, 1988b) menciona otro nombre para este sitio: Nohchén.

${ }^{7}$ Becquelin y Michelet reportan que el acceso al centro de Xculoc parece haber sido muy controlado; el conjunto del lado norte tiene una "puerta monumental" abovedada, que Pollock (1980: 382) ya había señalado.

${ }^{8}$ Sin embargo, la interpretación que la autora proporciona para este elemento arquitectónico difiere de la que Gallareta y su equipo de trabajo mencionan en el trabajo citado.

Estudios de Cultura Maya. Vol. XXI, 2000

Instituto de Investigaciones Filológicas/

Centro de Estudios Mayas, UNAM 


\section{ZOHCHÉN}

Detalle de la bóveda de acceso al conjunto formal (reconstrucción hipotética)

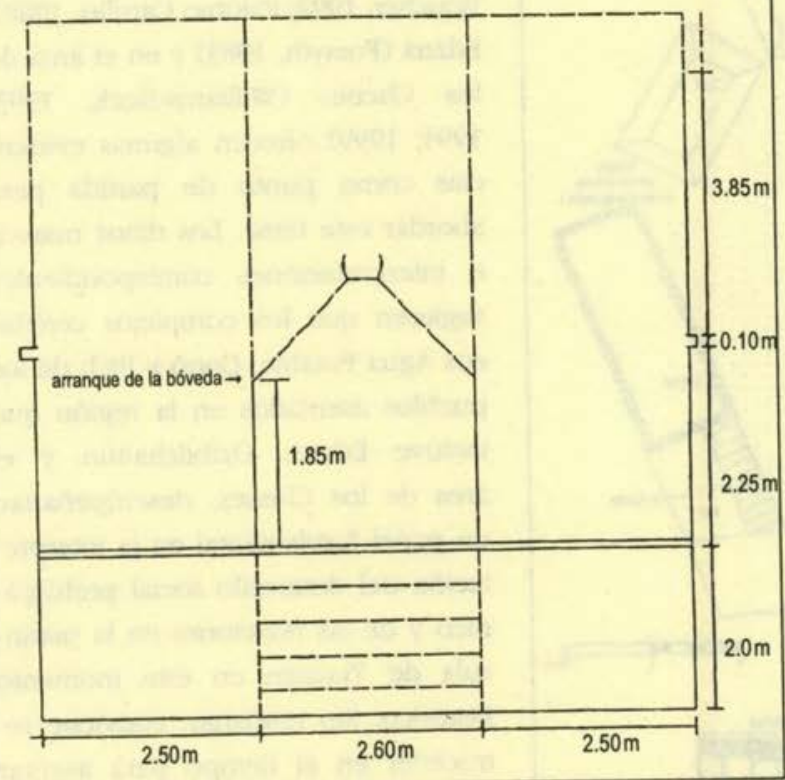

Figura 6. Detalle arquitectónico de la bóveda de acceso en el conjunto de acceso restringido en Zohchén

Si bien los grupos de patio en Pakchén (Xpulyaxché), Dzehkabtún y Dzibiltún continúan un patrón de diseño para estos conjuntos, sus elementos muestran también variaciones en torno del mismo concepto arquitectónico. Estos lugares carecen de una bóveda de acceso, pero muestran un espacio de entrada al conjunto entre dos montículos de menor tamaño. Asimismo cuentan con unos elementos ubicados en las esquinas noroeste-sureste, noreste-suroeste o en el costado norte, cuya forma en planta y perfil como "rampa" sugiere que tuviesen un acabado especial. En el caso de Dzibiltún, estos elementos cuentan con lajas reclinadas en el costado interior, que mira hacia el espacio compartido del patio (figura 7).

\section{La cerámica}

La cerámica proveniente de contextos de superficie o de excavación se considera tradicionalmente como la base no sólo para estimar el periodo de ocupación y actividad cultural en los sitios mayas, sino también para sugerir los vínculos que tenían con otros pueblos asentados en su alrededor. $\mathrm{Si}$ bien sus atributos de forma, acabado y producción proporcionan marcos temporales y culturales que permiten estimar la dinámica social, política y económica prehispánica en la península de Yucatán (Ball, 1993), estos procesos sopesaban anteriormente las tierras bajas del norte y del sur más que las del área central. Esto se debe a una labor de investigación arqueológica intensiva realizada en el extremo norte y oriente de la península de Yucatán (Andrews y Robles, 1986), en las áreas del Puuc (Smith, 1971) y Río Bec (Ball, 1977), en el valle de Belice (Gifford, 1976) y el área del Petén guatemalteco (Smith, 1955), soslayando la región centro-peninsular.

En este sentido, a partir de los años cuarenta los estudios cerámicos pioneros de Uaxactún (Smith, 1955) constituyeron la vara con la que se median las relaciones arquitectónicas y cerámicas entre los horizontes Formativo y Clásico Temprano, para después proponer algunas vías de crecimiento y desarrollo para los mayas prehispánicos. Las esferas de influencia derivadas de estos estudios (figura 8) presentaban un cuadro mediante el cual todas las relaciones entre los pueblos emanarían desde las tierras bajas de sur hacia sus pares del norte (Gendrop, 1983; Andrews, 1985). ${ }^{9}$ A partir del periodo Clásico Terminal esta

${ }^{9}$ En un trabajo inédito Boucher (1990) expone razones de peso sobre el desarrollo paralelo de las esferas cerá- 


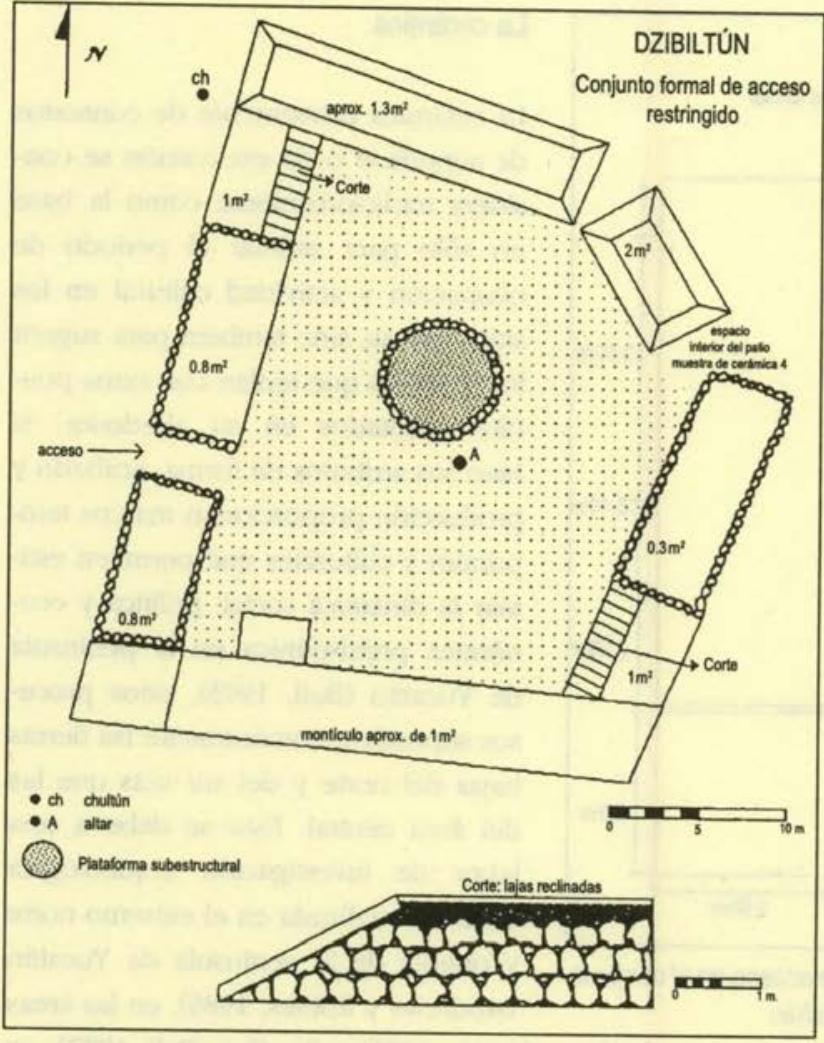

Figura 7. Plano del conjunto de acceso restringido en Dzibiltún

ruta de influencia cambiaría: los sitios del norte (Dzibilchaltún [Simmons, 1978-1980], Mayapán y Puuc [Smith, 1970]) formarían esta base de comparación. Si bien algunos de estos argumentos se han aceptado en lo general, entonces ¿en cuál zona se deberían buscar los antecedentes cerámicos para el periodo intermedio -el Clásico Tardío- que crearían unas técnicas de producción y acabado que serían retomadas en las esferas Cehpech y Sotuta del periodo Clásico Terminal-Posclásico Temprano? ${ }^{10}$

Investigaciones recientes sobre la cerámica del Clásico Tardío en el área centro-peninsular (Ball, 1977; Boucher, 1992; Palomo Carrillo, 1988), Edzná (Forsyth, 1983) y en el área de los Chenes (Williams-Beck, 1993, 1994, 1999) ofrecen algunas evidencias como punto de partida para abordar este tema. Los datos nuevos e interpretaciones correspondientes sugieren que los complejos cerámicos Agua Potable, Copó y Pich de los pueblos asentados en la región que incluye Edzná, Dzibilchaltún y el área de los Chenes, desempeñarían un papel fundamental en la interpretación del desarrollo social prehispánico y de las relaciones en la península de Yucatán en este momento histórico. Sin embargo, conviene retroceder en el tiempo para asentar las bases de este fenómeno.

Desde fines del periodo Clásico Temprano se pueden detectar algunos cambios tecnológicos y de producción para los complejos cerámicos del área centro-peninsular que darian impulso a la creación de nuevas vajillas cerámicas de producción regional (Ball, 1977; Brainerd, 1958; Boucher, 1992; Forsyth, 1983; WilliamsBeck, 1993, 1994, 1999), cuya influencia parece haberse difundido después en toda el área maya peninsular. Una de estas vajillas se reconoce por la decoración bicroma y policroma sobre un color de fondo anaranjado o de anaranjado sobre crema (Ball, 1975; Palomo Carrillo, 1988; Bou-

micas contemporáneas en las tierras bajas del norte y del sur durante los horizontes tempranos, que podría cambiar este cuadro de comparación.

${ }^{10} \mathrm{El}$ grado de traslape cronológico entre las esferas Cehpech y Sotuta ha sido un tema polémico con elementos de juicio a favor y en contra en los estudios recientes de las tierras bajas del norte. Por ser un tema que requiere una discusión amplia no será tratado en el presente trabajo. Sin embargo, el lector puede consultar los articulos de Anderson (1998), Andrews y Robles (1986) y Ball (1986) para tener mayor información al respecto. 
Figura 8. Cuadro de cronología comparativa

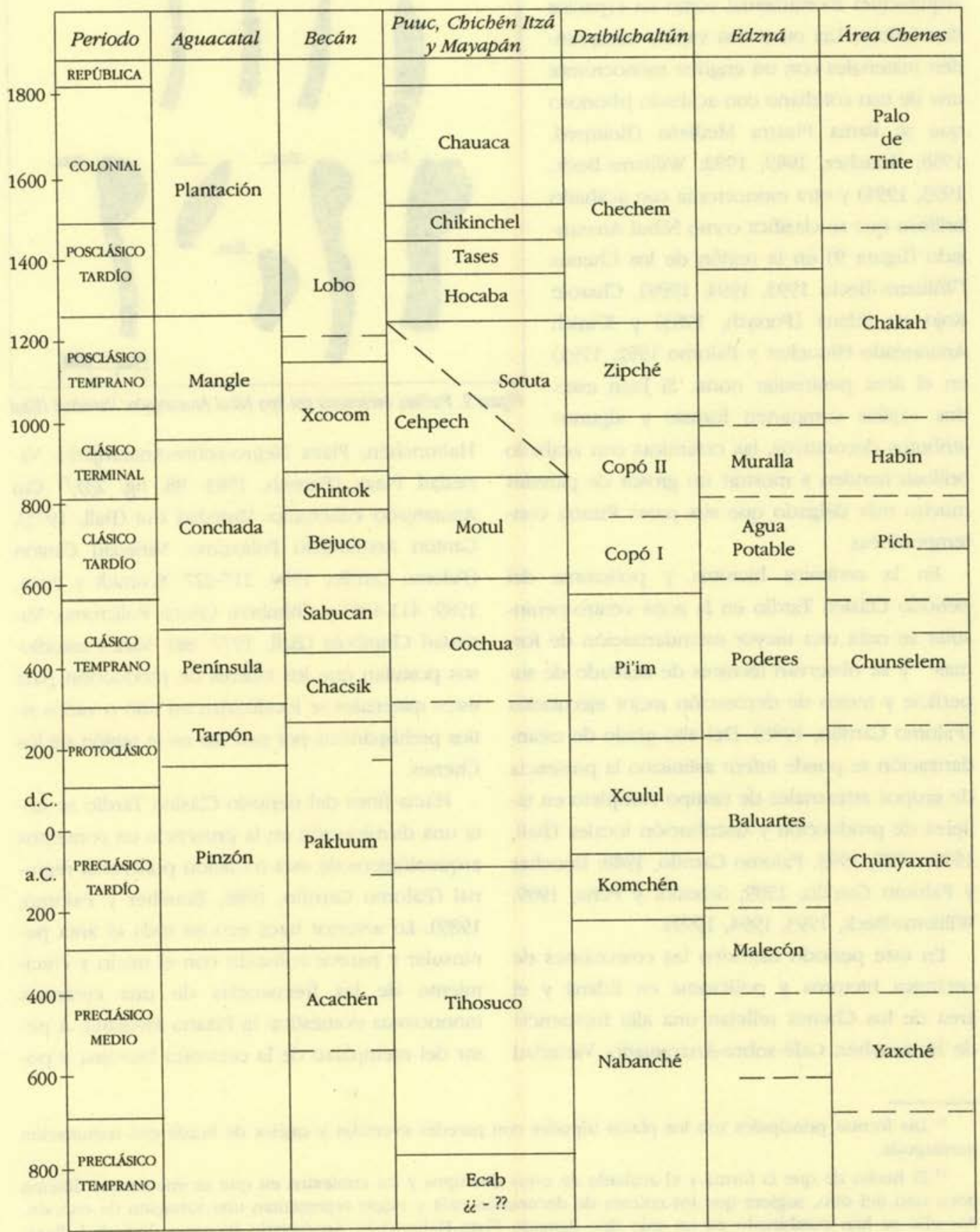

Estudios de Cultura Maya. Vol. XXI, 2000

Instituto de Investigaciones Filológicas/

Centro de Estudios Mayas, UNAM

ISSN: 0185-2574 
cher y Palomo, 1989; Schmidt y Peña, 1989) ha sido encontrada tanto en contextos de arquitectura monumental como en espacios domésticos. Las otras dos vajillas comprenden materiales con un engobe monocromo: una de uso cotidiano con acabado jabonoso que se llama Pizarra Mediana (Brainerd, 1958; Boucher, 1989, 1992; Williams-Beck, 1993, 1994) y otra monocroma con acabado brilloso que se clasifica como Nibul Anaranjado (figura 9) en la región de los Chenes (Williams-Beck, 1993, 1994, 1999), Charote Rojo en Edzná (Forsyth, 1983) y K'inich Anaranjado (Boucher y Palomo 1992, 1995) en el área peninsular norte. Si bien estas dos vajillas comparten formas y algunos atributos decorativos, las cerámicas con acabado brilloso tienden a mostrar un grosor de paredes mucho más delgado que sus pares Pizarra contemporáneas.

En la cerámica bicroma y policroma del periodo Clásico Tardío en la zona centro-peninsular se nota una mayor estandarización de formas $^{11}$ y se observan técnicas de acabado de superficie y trazos de decoración mejor ejecutados (Palomo Carrillo, 1988). Del alto grado de estandarización se puede inferir asimismo la presencia de grupos artesanales de tiempo completo en talleres de producción y distribución locales (Ball, 1975, 1977, 1993; Palomo Carrillo, 1988; Boucher y Palomo Carrillo, 1989; Schmidt y Peña, 1989; Williams-Beck, 1993, 1994, 1999).

En este periodo histórico las colecciones de cerámica bicroma y policroma en Edzná y el área de los Chenes reflejan una alta frecuencia de Haltunchén Café-sobre-Anaranjado: Variedad

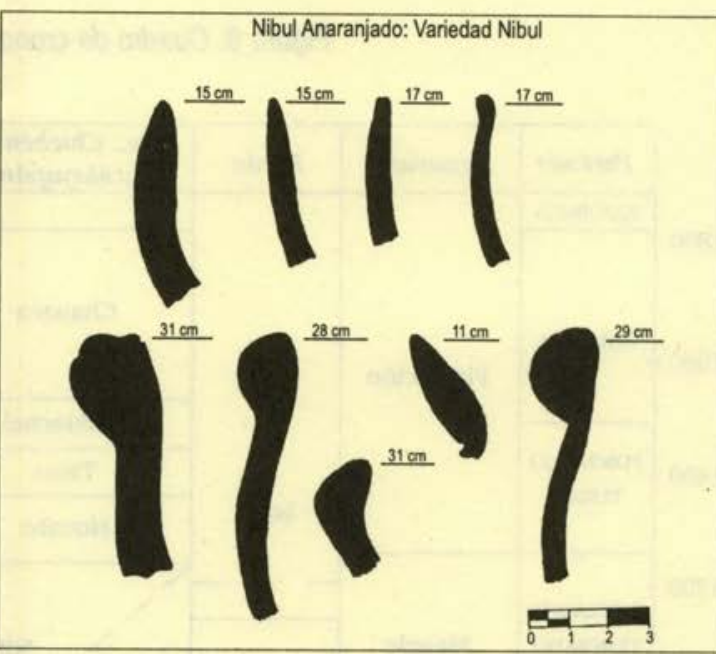

Figura 9. Perfiles cerámicos del tipo Nibul Anaranjado: Variedad Nibul Haltunchén, Plaza Negro-sobre-Anaranjado: Variedad Plaza (Forsyth, 1983: 88, fig. 23), ${ }^{12}$ Cui Anaranjado Policromo: Variedad Cui (Ball, 1975), Cantón Anaranjado Policromo: Variedad Cantón (Palomo Carrillo, 1988: 217-227; Schmidt y Peña, 1989: 411-426) y Chimbote Crema Policromo: Variedad Chimbote (Ball, 1977: 88). Varios estudiosos postulan que los talleres de producción para estos materiales se localizarían en uno o varios sitios prehispánicos por precisar en la región de los Chenes.

Hacia fines del periodo Clásico Tardío se nota una disminución en la presencia en contextos arqueológicos de esta tradición policroma regional (Palomo Carrillo, 1988; Boucher y Palomo, 1989). Lo anterior hace eco en toda el área peninsular y parece coincidir con el inicio y crecimiento de las frecuencias de una cerámica monocroma doméstica: la Pizarra Mediana. A pesar del reemplazo de la cerámica bicroma y po-

\footnotetext{
${ }^{11}$ Las formas principales son los platos trípodes con paredes evertidas y cajetes de borde con terminación puntiaguda.

${ }^{12} \mathrm{El}$ hecho de que la forma y el acabado de estos dos tipos y los contextos en que se encuentran difieran poco uno del otro, sugiere que los colores de decoración café y negro representan una variación de cocción. Por ello se han combinado en un solo tipo llamado Plaza-Haltunchén Anaranjado Bicromo: Variedad PlazaHaltunchén (Williams-Beck, 1993; 63-64) 2000
} 
licroma en la zona peninsular, los nuevos tipos de Pizarra continúan utilizando el repertorio de formas comunes entre sus pares brillosos, como los cajetes semiesféricos y los platos o cajetes con soporte anular o de trípode con forma cónica y maciza (Williams-Beck, 1993, 1994, 1999). Este hecho sugiere que ambas tradiciones de forma, acabado y decoración podrían coexistir en un área geográfica para su producción, quizá reflejando distintas escuelas de patrocinio. ${ }^{13}$ Asimismo las cerámicas brillosas de esta época parecen establecer algunos patrones morfológicos y tecnológicos que serían duplicados en los materiales Pizarra Mediana, tanto contemporáneos como aquellos que continuarian en uso durante el periodo Clásico Terminal en la península yucateca.

La coexistencia de tradiciones cerámicas en el área peninsular no es una hipótesis nueva. Brainerd (1958) sugiere esta posibilidad de variaciones temporales y/o geográficas en la cerámica Pizarra de los horizontes Clásico Tardío y Clásico Terminal. Hace poco, Boucher (1992) trazó el inicio y subsecuente desarrollo de estas vajillas monocromas domésticas a partir de los periodos Clásico Temprano y Clásico Tardío en el área centro-peninsular, incluyendo las zonas Río Bec, Chenes y Puuc. La identificación de estas vajillas del grupo cerámico Unpelchén (Clásico Tardío) en la región de los Chenes (Williams-Beck, 1993, 1994, 1999) ahora permite corroborar la hipótesis inicial de Brainerd sobre estos materiales Pre-Pizarra o Pizarra Proto-Cehpech. En cuanto a las formas y los acabados que sustentan, aquellas $\mathrm{Pi}$ zarras tempranas en el área de los Chenes ${ }^{1 /}$ difieren más de sus pares puucoides del grupo cerámico Muna Pizarra (Smith, 1971) que los asociados con el grupo cerámico Kapelchén Pizarra para el área Chenes (Williams-Beck, 1993, 1994, 1999).

Ahora bien, aquella técnica de decoración de un engobe doble (con color de fondo anaranjado o de anaranjado sobre crema), utilizada en los acabados bicromo $\mathrm{y}$ policromo de las vajillas Yucatán, Petén y Campeche Brillosas, se combina asimismo con otros modos de decoración en la cerámica monocroma de los grupos cerámicos Dzilam (Simmons, 1978-1980) o K'inich Anaranjado (Boucher y Palomo, 1995) en Dzibilchaltún y en sitios del área peninsular norte, el grupo Charote Rojo en Edzná (Forsyth, 1983: 9195) y el Nibul/K'inich Anaranjado en la región de los Chenes (Williams-Beck, 1993, 1994, 1999). Las cerámicas en estos grupos no sólo comparten un acabado brilloso de color anaranjado a anaranjado rojizo, sino también sugieren una variación en torno de una tradición cerámica local compartida entre el área peninsular central y el norte.

Coincidian Simmons (1978-1980), Forsyth (1983), Carrasco y Boucher (1990), Boucher y

\footnotetext{
${ }^{13}$ Sobre la base de estudios arqueológicos realizados en Belice, Ball (1993, pp. 20-27) plantea dos modos distintos de producción para la cerámica: la policroma o fina de uso especial parece haber sido producida por "escuelas palacio" con el patrocinio de grupos dirigentes en sitios o áreas específicas; mientras los materiales de uso cotidiano, como las vajillas estriadas sin engobe y otras con técnicas de manufactura y acabados menos complejos, corresponden a una tradición de producción por diversas "aldeas o comunidades" locales en una región inmediata.

${ }^{14}$ La cerámica Pizarra Mediana del periodo Clásico Tardío en la región de los Chenes muestra formas propias, como cazuelas de borde engrosado con el interior cóncavo, ollas con cuello cilíndrico cóncavo y algunos con borde en forma de gancho, cajetes con base anular (Boucher, 1992) y platos trípodes con paredes muy evertidas (Williams-Beck, 1993, 1994, 1999). Las ollas chultuneras fechadas a esta época suelen tener un engobe de acabado mate sobre una decoración estriada, o sin estrías y con un acabado menos translúcido y jabonoso al tacto. En cambio, la superficie interior de cazuelas, platos y cajetes lleva un engobe de acabado mate o jabonoso translúcido sobre el interior de la vasija que se extiende hasta el labio del borde exterior y unos centímetros abajo (Boucher, 1992; Williams-Beck, 1993, 1994, 1999).
} 
Palomo (1992, 1995) y Williams-Beck (1994) en el hecho de que los grupos cerámicos Charote, Dzilam o K'inich Anaranjado y Nibul/K'inich Anaranjado fuesen los posibles precursores a los materiales de la vajilla Puuc Rojo en el Complejo Cehpech del periodo Clásico Terminal en el área Puuc (Smith, 1971, vol. 1: 27, 156-158). A varios años de presentar estos comentarios en foros académicos distintos, considero que el grupo Nibul/K'inich Anaranjado del área de los Chenes podría ser asimismo una cerámica que precede a los tipos Tienda Pizarra Delgado y Teabo Rojo, por una correspondencia estrecha entre las formas que presentan. Por ello, se considera también que estas cerámicas de acabado anaranjado brilloso en el área de los Chenes podrian marcar un periodo de transición entre los horizontes Pich (Clásico Tardío) y Habín (Clásico Terminal), en virtud de esta relación en los repertorios formales.

A pesar del excelente estudio de contextos estratigráficos en Edzná, se plantea una afinidad cultural errónea para los tipos cerámicos del periodo Clásico Tardío Carpizo Rojo: Variedad Carpizo y Agua Potable Especial: Variedad Pizarra Puuc? (sic) y el Acapulquito Sin Engobe: Variedad Acapulquito ${ }^{15}$ del siguiente periodo Clásico Terminal (Forsyth, 1983: 95, 98, 124 y 125). Sobre la base de información que aporta el presente trabajo, ahora es posible ofrecer comentarios adicionales sobre la naturaleza de estas relaciones. El análisis minucioso de la colección cerámica para la región de los Chenes permite identificar frecuencias amplias y distribuciones considerables de la cerámica Pizarra Mediana del periodo Clásico Tardío y de las vajillas monocroma, bicroma y policroma de producción local en toda el área de estudio. Estos ma- teriales aparecen asimismo en la colección de Edzná (Williams-Beck, 1993, 1994, 1999). Merece revalorizarse también el hecho que la réplica entre formas y acabados para los tipos cerámicos que ocurren en la zona centro-peninsular parecen continuar en esta región hasta fines del periodo Clásico Terminal.

\section{Comentarios finales}

Los avances recientes en las lecturas de monumentos esculpidos sugieren algunas diferencias también entre los mayas asentados en el norte de la península, aquellos que residían en Edzná y los habitantes de la región de los Chenes, comparados con los grupos oriundos de las tierras bajas mayas del sur (Proskouriakoff, 1950; Grube, 1994). Si bien el contenido de monumentos encontrados en el área centro-peninsular no sugiere dinastías de familias como en las estelas de las tierras bajas del sur, las lecturas hablan sobre personajes destacados en Xcalumkin (Grube, 1994), Oxkintok (García Campillo, 1992), Uxmal y Chichén Itzá (Grube, 1994). Asimismo Grube (1994) ha identificado otra correlación para la región centro-peninsular, cuyo origen parece trazarse desde las fechas 9.12.0.0.0., es decir alrededor del año 700 de nuestra era. Sin duda alguna, este periodo histórico sería un momento clave en el futuro desarrollo cultural del área.

El análisis breve de los elementos arquitectónicos y cerámicos presentado en este ensayo, así como la distribución de estos materiales culturales en su contexto geográfico y temporal, sugiere algunas interpretaciones nuevas para el área centro-peninsular a partir del periodo Clá-

${ }^{15}$ El tipo Acapulquito Sin Engobe: Variedad Acapulquito ha sido reclasificado por mí en el periodo Clásico Tardío como tipo Acapulquísimo Café:Variedad Acapulquísimo (Williams-Beck, 1993: 46; 1999: 84-85 y fig. 15xdd), pues muestra todos los indicadores de producción y acabado señalados para el material Pizarra Temprano (Boucher comunicación personal, 1992) 2000

Instituto de Investigaciones Filológicas/

Centro de Estudios Mayas, UNAM

ISSN: 0185-2574 
sico Tardío y hasta el Clásico Terminal. El intercambio que se puede inferir a través de las evidencias arqueológicas y arquitectónicas en su marco histórico cultural daría impulso a ciertas respuestas específicas manifestadas en el proceso de su propio desarrollo.

Por un lado, la arquitectura y los conjuntos de patio con acceso restringido muestran una variación en torno de un concepto genérico arquitectónico, que fue compartido por estos lugares durante periodos específicos en su historia. Asimismo, las réplicas arquitectónicas no son elementos aislados: el contenido cerámico en estos contextos sugiere otra réplica estructural por la forma, frecuencia, distribución y el contenido de las vajillas monocromas y policromas encontradas de un sitio a otro. La duplicidad de patrones en estos contextos sugiere una relación estrecha de intercambio de conceptos e ideas básicos entre los antiguos moradores del área centro-peninsular.

Por otra parte, las colecciones cerámicas contemporáneas en Edzná y en la región de los Chenes (Forsyth, 1983; Williams-Beck, 1993, 1994, 1999), compartidas con algunos tipos cerámicos identificados en Dzibilchaltún (Simmons, 1978-1980), sugieren una zona de interacción estrecha entre algunos sitios del norte y del centro de la península a partir del periodo Clásico Tardío. Esta información permite plantear una nueva esfera de relaciones entre arquitectura, cerámica y espacios culturales, cuyos socios serían el área de los Chenes, Edzná, Jaina, la costa del Golfo y Dzibilchaltún en el norte (WilliamsBeck, 1993, 1994, 1999).

Por último, el gran apogeo cultural en la zona peninsular norte que empezó a finales del periodo Clásico Terminal y a principios del Posclásico Temprano debería reflejar el cúmulo de algunas relaciones históricas y culturales anteriores a esto. Estos vínculos pudieran haber asentado las bases previas al desarrollo social, político y económico tan notorio en esta zona más tarde. Dadas las circunstancias históricas en torno a una relación muy cercana entre réplicas estructurales arquitectónicas y respuestas cerámicas encontradas en Edzná y en la región de los Chenes, es probable que el producto de estas relaciones pueda haberse plasmado en otras condiciones políticas $y / 0$ sociales distintas para esta zona, de las que aún se espera esclarecer mayores detalles sobre su intensidad y naturaleza. Un posible resultado de estas condiciones particulares proporcionaría otra serie de réplicas estructurales, políticas y culturales, que se verían más tarde en sitios como Uxmal, Chichén Itzá, Mayapán y la costa del Caribe y perdurarían en fechas posteriores al abandono parcial y/o completo de la región centro-peninsular.

\section{Obras citadas}

Anderson, Patricia K.

1998 "Yula, Yucatán, México: Terminal Classic Maya Ceramic Chronology for the Chichen Itza Area", Ancient Mesoamerica, 9 (1): 151-166.

Andrews, George F.

1975 Maya Cities: Placemaking and Urbanization, University of Oklahoma Press, Norman.

1984 Edzna, Campeche, Mexico: Settlement Patterns and Monumental Architecture, Foundation for Latin American Anthropological Research, Culver City, California.

1985 "Chenes-Puuc Chronology and Cultural Interaction", en Arquitectura y arqueologia: metodologías en la cronología de Yucatán, свмса, México, pp. 11-40.

1988a "Recent Discoveries at Two Chenes Archaeological Sites in Mexico", Mexicon, X, 4, pp. 70-77.

1988b "Additions and Corrections", Mexicon, X, 6, p. 111.

BALL, JOSEPH W.

1974 "A Regional Ceramic Sequence for the Rio Bec Area", en Archaeological Investigations on the Yucatan Peninsula, Tulane University Publication 31, Nueva Orleans, pp. 113-117. 
1975 “Cui Orange Polychrome: a Late Classic Funerary Type from Central Campeche, Mexico", en Studies in Ancient Mesoamerica, Publication 27, pp. 32-39, Archeological Research Facility, Berkeley.

1977 The Archeological Ceramics of Becan, Campeche, Mexico, Publication 43, Tulane University, Nueva Orleans.

1978 "Archaeological Pottery of the Yucatan-Campeche Coast", en J. D. Eaton y J. W. Ball, Studies in the Archaeology of Coastal Yucatan and Campeche, Mexico, Nueva Orleans, Publication 46, pp. 69-146, Middle American Research Institute.

1985 "The Postclassic Archeology of the Western Gulf Coast: Some Initial Observations", en A. F. Chase y P. M. Rice (eds.), The Lowland Maya Postclassic, University of Texas Press, Austin.

1993 "Pottery, Potters, Palaces, and Polities: Some Socioeconomic and Political Implications of Late Classic Maya Ceramic Industries", en J. A. Sabloff y J. S. Henderson (eds.), Lowland Maya Civilization in the Eightb Century A.D., Dumbarton Oaks, Trustees for Harvard University, Washington, D.C., pp. 243-272.

Benavides C., Antonio

1997 Edzná: un sitio prebispánico en Campeche, Mexico / Edzná: a pre-Hispanic site in Campeche, Mexico. INAH, Colección científica y University of Pittsburgh Press, México y Pitssburgh.

BOUCHER, SylviAnE

1990 "Algunos comentarios sobre la cerámica de los horizontes Formativo y Floreciente en las áreas Puuc y Río $\mathrm{Bec}^{\prime \prime}$, ponencia presentada en el Primer Simposio Maler sobre la Arqueología del Noroeste de Yucatán, Bonn, Alemania, agosto.

1992 "Cerámica Pizarra Temprana: algunas precursores y variantes regionales", Memorias del Primer Congreso Internacional de Mayistas, UNAM, IIF-CEM, México, pp. 464-476.

Boucher, Sylviane, y Yoly Palomo Carrilo

1989 "Estilo regional en cerámica policroma de Campeche", Memoria del Segundo Coloquio Internacional de Mayistas, UNAM, IIF-CEM, México, pp. 485-516.

1995 “El grupo K'iich Naranja: Un sistema ceráEsmico delcGlásiconTardípoenxel nomoeste de la Instituto de Investigaciones Filológicas/

Centro de Estudios Mayas, UNAM

ISSN: 0185-2574 península de Yucatán", Memorias del Segundo Congreso Internacional de Mayistas, tomo I pp. 464-476, UNAM, ПF-Cem, México.

Brainerd, George W.

1958 The Archeological Ceramics of Yucatan, Anthropological Records 19, University of California Press, Berkeley.

Becquelin, Pierre, y Dominique Michelet

1988 Informe $3^{\text {a }}$ Temporada [en Xculoc], Archivo Técnico de Prehispánicos-INAH, vol. IX, Estado de Campeche, 1983-1989, pp. 3-22.

Carrasco, Ramón, y Sylviane Boucher

1985 "Nuevas perspectivas para la cronología y el estudio de la arquitectura de la Región Central de Yucatán", en Arquitectura y arqueología: Metodologías en la cronología de Yucatân, семсл, México, pp. 57-68.

1986 "Comentarios a dos artículos recientes publicados en Cuadernos de Arquitectura Mesoamericana", Cuadernos de Arquitectura Mesoamericana, núm. 7, pp. 92-94.

1990 "El palacio de Sayil (estr. 2B1): un estudio cronológico", en Mesoamérica y el norte de México, siglos IX al XII, INAH-Museo Nacional de Antropología, México, pp. 59-85.

FORSYTH, DONALD W.

1983 Investigations at Edzna, Campeche, Mexico, vol. 2: Ceramics, New World Archeological Foundation Publication 46, Utah.

García Cruz, Florentino

1989 "Arquitectura petén en Edzná, Campeche: temporada de campo 1987", en Memorias del Segundo Coloquio Internacional de Mayistas, UNAM, III-CEM, México, pp. 523-530.

Gendrop, PAUL

1983 Los estilos Río Bec, Cbenes y Puuc en la arquitectura maya, unam, Facultad de Arquitectura, México.

Gallareta Negrón, Tomís, et al.

[1993] "Investigaciones arqueológicas recientes en Labná, Yucatán", ponencia presentada en la XX Mesa Redonda de Palenque, Palenque, Chiapas, en julio de 1993.

Garcia Campilo, José Miguel.

1992 "Informe epigráfico sobre Oxkintok y la cerámica Chochola" en Miguel Rivera Dorado et al., Oxkintok, vol. 4, pp. 185-200, Ministerio de Cultura de España, Madrid.

Grube, Nicolal

1994 "Hieroglyphic Sources for the History of 
Northwest Yucatan", en Hanns J. Prem, (ed.), Hidden Among the Hills: Maya Archaeology of the Northwest Yucatan Peninsula, Verlag Von Flemming, Möckmühl, pp. 316-358.

KURJACK, EDWARD B.

1975 Prebistoric Lowland Maya Community and Social Organization, Nueva Orleans, Tulane University, Middle American Research Institute Publication 38.

1994 "Political Geography of the Yucatecan Hill Country", en Hanns J. Prem (ed.), Hidden Among the Hills: Maya Archaeology of the Nortbwest Yucatan Peninsula, Verlag Von Flemming, Möckmühl, pp. 308-315.

Maldonado Cárdenas, Rubén

[1993] Nuevas exploraciones en Dzibilchaltún, ponencia presentada en la XX Mesa Redonda de Palenque, Palenque, Chiapas, en julio de 1993.

Miluet Cámara, Luis

1989 "Edzná, Campeche: una revaloración de su historia", en Memorias del Segundo Coloquio Internacional de Mayistas, UNAM, IIF-CEM, México, pp. 517-521.

[1990] El juego de pelota de Edzná, ponencia presentada en la Segunda Jornada de la Historia de Campeche, Instituto de Cultura de Campeche, del 5 al 7 de octubre de 1990.

Palomo Carrillo, Yoly Esperanza

1988 Catâlogo de vasijas policromas mayas: diferencias estilísticas regionales, tesis profesional de licenciatura en ciencias antropológicas, Universidad Autónoma de Yucatán.

Piña Chan, Román

1975 Edzná: guía de la zona arqueológica, INAH, México.

1985 Cultura y ciudades mayas de Campeche, Editora del Sureste, México.

PotTer, DAvid F.

1977 Maya Architecture of the Central Yucatan Peninsula, Mexico, Tulane University, Middle American Research Institute Publication 44, Nueva Orleans,

Press, Irwin, y M. Estelue Smith

1980 Urban Place and Process. Readings in the Antbropology of Cities, MacMillan Publishing, Nueva York.
Proskouriakoff, TATIANA

1950 Classic Maya Inscriptions, Peabody Museum and Harvard University Press, Cambridge.

Robles Casteluanos, José Fernando

1990 La secuencia de la cerámica de la región de Cobá, Quintana Roo, INAH-SEP, Colección Científica, 184, México.

RUz LHUILIER, AlBerto

1945 "Campeche en la arqueología maya", Acta Antropológica, 2-3, México.

Schmidt, Peter, y Agustín Peña

1989 "Cantón Naranja Policroma: un tipo cerámico de los Chenes", Memorias del Segundo Coloquio Internacional de Mayistas, UNAM, IIFCEM, México, pp. 411-426.

Simmons, Michael. D.

1978 The Post-Formative Ceramics of Dzibilchaltún, Yucatán, México, copia fotostática del manuscrito inédito se encuentra en la Ceramoteca del CRY-INAH.

SMITH, ROBERT E.

1955 Ceramic Sequence of Uaxactun, Middle American Research Institute Publication 26, Nueva Orleans,

1971 The Pottery of Mayapan, Including Studies of Ceramic Material from Uxmal, Kabah and Chichen Itza, Papers of the Peabody Museum of Archeology and Ethnology 66, Cambridge.

WILLAMs-Beck, LoRraine A.

1993 Tierra de nadie: cerâmica, arquitectura y sociedad prebispánica en la región Chenes, Campeche, México, tesis de doctorado en investigación antropológica, Unidad Académica de los Ciclos Profesional y de Posgrado del CCH, UNAM.

1994 "The Chenes Ceramic Sequence: Temporal, Typological, and Cultural Relations in a Regional Framework", en Hanns J. Prem (ed.), Hidden Among the Hills: Maya Archaeology of the Northwest Yucatan Peninsula, Verlag Von Flemming, pp. 133-165, Möckmühl.

1999 Tiempo en trozos: Cerámica de la región de los Chenes, Campeche, México, Universidad Autónoma de Campeche, Gobierno del Estado de Campeche, Instituto de Cultura de Campeche / Fondo Estatal para la Cultura de Campeche / cnca, Campeche. 